\title{
Remimazolam Has Low Oral Bioavailability and No Potential for Misuse in Drug-Facilitated Sexual Assaults, with or Without Alcohol: Results from Two Randomised Clinical Trials
}

\author{
Marija Pesic $^{1}$ (D) $\cdot$ Thomas Stöhr $^{1} \cdot$ Joachim Ossig ${ }^{1} \cdot$ Keith Borkett $^{1,2} \cdot$ Martin Donsbach $^{1} \cdot$ Van-Anh Dao $^{1}$. \\ Lynn Webster ${ }^{1,3} \cdot$ Frank Schippers $^{1,4}$
}

Published online: 5 August 2020

(c) The Author(s) 2020

\begin{abstract}
Background and Objectives Remimazolam is a new ultra-short-acting benzodiazepine currently being developed for intravenous use in procedural sedation, general anaesthesia, and intensive care unit sedation. Benzodiazepines represent a drug class associated with drug-facilitated sexual assaults, especially in combination with alcohol. Two clinical trials were designed to evaluate the oral bioavailability and pharmacokinetics/pharmacodynamics of remimazolam and to assess the potential for remimazolam misuse in drug-facilitated sexual assaults via oral ingestion.

Methods Trial 1 was conducted in 14 healthy volunteers to evaluate the oral bioavailability of remimazolam. Part 1 of trial 2 was conducted in 21 healthy female volunteers to find the minimal biologically active dose of oral remimazolam. Part 2 of trial 2 was conducted in 11 healthy female volunteers to evaluate the pharmacokinetics/pharmacodynamics of oral remimazolam in combination with alcohol.

Results Remimazolam undergoes rapid and extensive first-pass metabolism upon oral administration. The oral bioavailability of remimazolam was negligible $(2.2 \%$ based on total systemic exposure and $1.2 \%$ based on maximum plasma concentration). Plasma clearance of both remimazolam and its metabolite was fast (elimination half-life 20-40 min and 1.75-2 h, respectively). Alcohol did not appear to inhibit the rapid first-pass metabolism of remimazolam. No clear sedative effects were observed for remimazolam without alcohol. Significant sedation was observed in one of ten subjects after remimazolam $360 \mathrm{mg}$ (18 drug product vials) $+40 \%$ v/v alcohol.

Conclusion The oral bioavailability of remimazolam is negligible, which-together with its distinct bitter taste-suggests no meaningful potential for misuse in drug-facilitated sexual assaults via oral ingestion, with or without alcohol.

Clinical Trial Registration Numbers Trial 1 (NCT04113564) and trial 2 (NCT04113343) both retrospectively registered on 2 October 2019.
\end{abstract}

Joachim Ossig: Deceased.

Electronic supplementary material The online version of this article (https://doi.org/10.1007/s40268-020-00317-0) contains supplementary material, which is available to authorized users.

Marija Pesic

m.pesic@paion.com

1 PAION Deutschland GmbH, Martinstr. 10-12, 52062 Aachen, Germany

2 Present Address: Carden House, Meadow Lane, Houghton PE28 2BP, Cambs, UK

3 Early Development Services, Scientific Affairs, PRA Health Sciences, Salt Lake City, UT, USA

4 Creative Clinical Research - CCR GmbH, Wallenroder Straße 7-9, 13435 Berlin, Germany

\section{Key Points}

Remimazolam has very low oral bioavailability of $1.2-2.2 \%$.

Oral remimazolam up to $480 \mathrm{mg}$ has no clear pharmacodynamic effects.

Alcohol dose-dependently increases exposure to remimazolam (up to two times).

Oral co-administration of alcohol and remimazolam shows mild pharmacodynamic effects.

Remimazolam has no potential for misuse in drug-facilitated sexual assaults. 


\section{Introduction}

Remimazolam is a new ultra-short-acting benzodiazepine being developed for intravenous procedural sedation, general anaesthesia, and sedation in the intensive care unit [1]. Benzodiazepines exhibit depressant properties on the central nervous system (CNS), resulting in psychomotor effects such as sedation, including the loss of consciousness and anterograde amnesia [2]. Such psychomotor effects pose the risk of benzodiazepines being misused in drug-facilitated sexual assaults, which usually happens after time spent in a party or a bar, and involves higher levels of drinking and selfreported intoxication with alcohol [3]. Toxicological data from victims, primarily women, showed that benzodiazepines are among the most common CNS-depressant drugs used in drug-facilitated sexual assaults and are found in the blood and urine of $\sim 30 \%$ of cases across the USA, following alcohol and cannabis [4, 5]. Long- and intermediate-acting orally administered benzodiazepines such as flunitrazepam and diazepam are the most commonly used, followed by temazepam, lorazepam, and nitrazepam [6].

When co-ingested orally with alcohol, the psychomotor effects of many benzodiazepines are potentiated [7, 8], making this combination even more dangerous and prevalent in drug-facilitated sexual assaults. This potentiating effect is mediated via two different mechanisms. First, both alcohol and benzodiazepines bind to distinct binding sites on the $\mathrm{GABA}_{\mathrm{A}}$ receptor, ultimately leading to synergistic drug actions [9]. Moreover, like alcohol, many benzodiazepines are metabolised by cytochrome P450 enzymes [10], resulting in substrate competition and delayed clearance of benzodiazepines when co-ingested orally with alcohol [11]. Unlike other benzodiazepines, the conversion of remimazolam to its inactive metabolite is mediated by liver carboxylesterases 1A (CES-1A), with no contribution of cytochrome P450 enzymes. Nonetheless, alcohol is known to inhibit CES-1 function and can alter the disposition, efficacy, and safety of drugs metabolised by CES-1 [12]. Thus, potential exists for remimazolam's sedative effect to be potentiated by alcohol via two distinct mechanisms, as is the case with other benzodiazepines. Therefore, a theoretical potential also exists for its misuse, alone or in combination with alcohol, in drugfacilitated sexual assaults.

We report here the results of two clinical trials designed to evaluate the bioavailability and pharmacokinetic/pharmacodynamic (PK/PD) profile of oral remimazolam, alone and in combination with alcohol, and to investigate the potential for misuse of oral administration of remimazolam in, for example, drug-facilitated sexual assaults.

\section{Materials and Methods}

\subsection{Trial 1: Oral Bioavailability of Remimazolam}

\subsubsection{Trial Design}

This randomised, open-label, phase I trial was conducted to determine the absolute oral bioavailability of remimazolam. A total of 14 healthy volunteers (at least five of each sex) aged 18-55 years, weighing $\geq 50 \mathrm{~kg}$ with a body mass index (BMI) of 18.0-32.0 kg/m ${ }^{2}$ were recruited. Each subject received oral remimazolam $0.14 \mathrm{mg} / \mathrm{kg}$ and intravenous remimazolam $0.025 \mathrm{mg} / \mathrm{kg}$ under fasting conditions in a cross-over design, allowing for a washout period of at least $24 \mathrm{~h}$ between administrations. Volunteers were asked to abstain from alcohol, caffeine, and xanthine-containing beverages or food (e.g. coffee, tea, cola, chocolate, energy drinks) during the trial.

The intravenous dose of $0.025 \mathrm{mg} / \mathrm{kg}$ was selected because it produced measurable plasma concentrations of remimazolam and its main metabolite CNS7054 but was devoid of sedative effects [13]. The oral dose was selected based on the expectation of oral bioavailability to be between $\sim 10 \%$ (results of non-clinical study in rabbits) and, as a worst-case assumption, $100 \%$. Since $0.28 \mathrm{mg} / \mathrm{kg}(20 \mathrm{mg}$ intravenous bolus in a person weighing $70 \mathrm{~kg}$ ) was considered the highest safe intravenous dose and hence the highest oral dose to be given in case of $\sim 100 \%$ bioavailability, $0.14 \mathrm{mg} / \mathrm{kg}$ (half that dose), was selected as a precautionary measure.

\subsubsection{Assessments}

Blood samples for pharmacokinetic assessments ( $2 \mathrm{~mL})$ were taken at pre-determined timepoints: pre-dose, 2, 5, 15,30 , and $45 \mathrm{~min}$ and $1,1.5,2,3,4,6,8$, and $12 \mathrm{~h}$ postdose using an intravenous line dedicated for pharmacokinetic sampling. Bioanalysis of remimazolam (method range 2-2000 ng/mL) and CNS7054 (method range 20-20,000 ng/ $\mathrm{mL}$ ) in human plasma was carried out via high-performance liquid chromatography analyses with tandem mass spectrometry detection using a validated bioanalytical method. Linearity (correlation coefficient) of the method was $>0.9963$ for remimazolam and 0.9964 for CNS7054. Within-run precision was $<6.6 \%$ and $<5.1$, and accuracy was 99.7-113.6 and 85.2-107.7 for remimazolam and CNS7054, respectively. Pharmacokinetic variables were derived from the plasma concentrations and non-compartmental analyses of plasma concentration-time data for remimazolam and its metabolite 
(CNS7054). Safety measurements, including adverse events, were documented. Demographic data (age, sex, race, and ethnicity) and medical histories were collected at screening.

The oral bioavailability determined in this trial was used to guide dose selection in trial 2.

\subsection{Trial 2: Pharmacokinetic/Pharmacodynamic (PK/PD) Effects of Oral Remimazolam with and Without Alcohol}

This two-part trial investigated the PK/PD effects of higher doses of oral remimazolam in healthy female volunteers aged $21-45$ years, weighing $\geq 50 \mathrm{~kg}$ with a BMI of $18-33 \mathrm{~kg} /$ $\mathrm{m}^{2}$, and moderate drinking habits. These were defined as more than two drinks/week and $\leq 14$ drinks/week (one drink equals approximately $12 \mathrm{oz} / 350 \mathrm{~mL}$ of beer, $5 \mathrm{oz} / 150 \mathrm{~mL}$ of wine, or $1.5 \mathrm{oz} / 45 \mathrm{~mL}$ of spirits). Female subjects were selected because they represent the population at greatest risk for victimization in drug-facilitated sexual assaults. In addition, females have a slower alcohol metabolism, so the effects of remimazolam may last longer in the presence of alcohol. To ensure the absence of pharmacodynamic interactions with any other CNS depressant, apart from investigated alcohol in part 2 of the trial, volunteers who tested positive for opiates, methadone, cocaine, amphetamines (including ecstasy), cannabinoids, barbiturates, benzodiazepines, tricyclic antidepressants, and alcohol in drug and alcohol screening were excluded from the trial.

\subsubsection{Trial Design}

2.2.1.1 Part 1: Minimally Biological Active Dose of Oral Remimazolam This was an open-label, dose-ascending trial to find the minimal biologically active dose (MBAD) of oral remimazolam. Up to 21 healthy female volunteers were to be recruited and allocated to five sequential groups of ascending oral remimazolam doses: $60 \mathrm{mg}(n=3), 140 \mathrm{mg}$ $(n=3), 240 \mathrm{mg}(n=5), 360 \mathrm{mg}(n=5)$, and $480 \mathrm{mg}(n=5)$. A minimum 10-h fast was required before administration of trial drug.

MBAD, in the context of this trial, was defined as the lowest dose that affects consciousness and was identified using the modified observer's assessment of alertness/sedation (MOAA/S) scale from 0 (deeply sedated) to 5 (fully alert). MBAD was characterised by occurrence of MOAA/S $<3$ in at least two of three subjects in the $60 / 140 \mathrm{mg}$ dose groups or at least three of five subjects in the 240/360/480 mg dose groups.
Based on the oral bioavailability results from trial 1, $60 \mathrm{mg}$ was expected to be a safe dose without clear sedative effects so was used as the starting dose in the second trial. An oral dose of $480 \mathrm{mg}$ (corresponding to 24 vials of remimazolam $20 \mathrm{mg}$ drug product) was approximately equivalent to a pharmacologically active intravenous dose of $5 \mathrm{mg}$ and was defined as the maximal dose to be tested in trial 2. Upon each dose group was completed, a safety review committee (SRC), including the investigators, evaluated the available safety and pharmacodynamic data and decided whether it was safe to escalate to a higher dose. The dose-stopping criteria are described in electronic supplementary material (ESM) 1.

A dose of two levels below the MBAD was to be the starting dose in part 2, so the subjects' safety could be ensured in case the sedative effects of remimazolam were augmented by alcohol. If no MBAD was identified based on the results of part 1 , the doses used in part 2 were to be determined by the SRC but not to exceed $480 \mathrm{mg}$.

2.2.1.2 Part 2: Effects of Alcohol on the PK/PD Profile of Oral Remimazolam This was a randomised, double-blind, placebo-controlled trial to evaluate the effects of alcohol on the PK/PD of oral remimazolam. Up to three escalating doses of remimazolam were to be investigated; as in part 1 , the SRC was to make decisions about dose escalation (dose-stopping criteria are described in ESM 1). With each oral remimazolam dose, 12 healthy female volunteers were to be recruited. Subjects were randomised to a treatment sequence consisting of five treatments: remimazolam, remimazolam $+5 \%$ v/v alcohol, remimazolam $+15 \% \mathrm{v} / \mathrm{v}$ alcohol, remimazolam $+40 \% \mathrm{v} / \mathrm{v}$ alcohol, and placebo $+40 \%$ $\mathrm{v} / \mathrm{v}$ alcohol in a five-way cross-over design. A minimum 10-h fast and a minimum 24-h washout were scheduled between consecutive treatments to avoid possible carryover effects. Treatments were administered as a "first drink" (125 mL apple juice, cinnamon/apple cider with or without alcohol) and a "second drink" (50 mL water containing remimazolam or placebo and the bitter agent BITREX ${ }^{\circledR}$ to mask the remimazolam taste). The "first drink" was to be consumed within 5 min and the "second drink" immediately thereafter.

As results of part 1 did not reveal an MBAD, the SRC chose oral remimazolam $360 \mathrm{mg}$ as a starting dose for part 2. Upon completion of the investigation of the $360 \mathrm{mg}$ dose, the SRC decided not to escalate to $480 \mathrm{mg}$ because the risk would outweigh any potential benefit from dose escalation. The trial was therefore terminated. 
Both parts 1 and 2 comprised three trial visits: an outpatient screening visit, a 3-day (part 1) or 7-day (part 2) inpatient treatment visit, and a follow-up/end-of-trial visit conducted via telephone.

The design of trial 2 is summarized in a flow chart in the ESM.

\subsubsection{Assessments}

Pharmacokinetic assessments were conducted as described in trial 1 . The bioanalytical method used in trial 1 was revalidated for this trial. The main difference was the use of isopropyl alcohol instead of methanol as the precipitation agent.

Pharmacodynamic assessments included an alertness/ drowsiness visual analogue scale (VAS; $0=$ very drowsy, $100=$ very alert), the MOAA/S, a Paired Associates Learning Test (PAL), and a Reaction Time Test (RTI). The alertness/drowsiness VAS, PAL, and RTI were recorded using the software CANTAB (Cambridge Cognition). These pharmacodynamic parameters reflect psychomotor effects such as level of sedation, cognitive functions, and memory [14, 15].

As in trial 1, demographic data (ethnicity, age, BMI) were collected at screening. All subjects in part 1 and 2 were females.

Trial 1 was carried out in November 2015 and trial 2 from May to August 2017. Both trials were conducted in the PRA Early Development Services clinical site in Utah, USA. Both trials were carried out in accordance with The Code of Ethics of the World Medical Association (Declaration of Helsinki). The trial 1 protocol was approved by the Midlands Independent Review Board and the trial 2 protocol was approved by the Schulman Independent Review Board. Informed consents were obtained from all participants.

\subsection{Statistical Analyses}

The pharmacokinetic parameters for remimazolam and CNS7054 were estimated using non-compartmental methods with Phoenix ${ }^{\circledR}$ WinNonlin $^{\circledR}$, version 6.3. The plasma pharmacokinetic parameters were estimated from the concentration-time profiles for the pharmacokinetic population. The relative bioavailability of oral versus intravenous formulations of remimazolam was calculated using the ratio and $90 \%$ confidence interval (CI) of geometric means for maximum observed plasma concentration $\left(C_{\max }\right)$, area under the curve (AUC) $)_{0-t}$, and $\mathrm{AUC}_{0-\text { inf. }}$. Actual body weights of participants were used for calculating all pharmacokinetic parameters. Pharmacodynamic results and changes from pre-dose were summarised by treatment and scheduled time point using descriptive statistics.

\section{Results}

\subsection{Participants}

In trial 1, 14 of 30 screened healthy volunteers were enrolled. The majority of subjects were White $(n=13)$, one subject was American Indian or Alaska Native, and four were of Hispanic or Latino ethnicity. All subjects were included in the pharmacokinetic and the safety populations (Fig. S1 in the ESM). Enrolled participants were aged 20-52 years with equal sex distribution and a BMI ranging between 19.2 and $31.8 \mathrm{~kg} / \mathrm{m}^{2}$. Participants' weight ranged between 50 and $103.2 \mathrm{~kg}$.

In part 1 of trial 2, 21 healthy females were enrolled, the majority of whom were White $(n=20)$ and not of Hispanic or Latino ethnicity $(n=17)$. Two of these did not complete the trial but were not excluded from the PK/PD or safety population (one subject was lost to follow-up, and one subject withdrew consent, both after completion of dosing). In part 2, 11 healthy females were enrolled (all White and not of Hispanic or Latino ethnicity); one of those did not complete the trial (withdrew consent before completion of the last dosing) and was excluded from the pharmacodynamic population but not from the pharmacokinetic or safety population (Fig. S1 in the ESM). Participants' age ranged from 21 to 40 years in part 1 and from 25 to 41 years in part 2 with similar BMI $\left(19.4-32.6 \mathrm{~kg} / \mathrm{m}^{2}\right)$.

\subsection{Pharmacokinetics of Remimazolam}

Table 1 and Fig. 1 present the pharmacokinetic analyses of remimazolam and its inactive metabolite (CNS7054) as assessed in trial 1 (intravenous dose $0.025 \mathrm{mg} / \mathrm{kg}$ corresponding to $1.25-2.58 \mathrm{mg}$; oral dose $0.14 \mathrm{mg} / \mathrm{kg}$ corresponding to $7.00-14.4 \mathrm{mg}$ ) and in part 1 of trial 2 (oral doses 60, 140, 240, 360, and $480 \mathrm{mg}$ ).

The $C_{\max }$ was higher for intravenous than for all oral doses except the highest $(480 \mathrm{mg}$ ), which had reached plasma levels comparable to intravenous doses $(92.9$ vs. $94.6 \mathrm{ng} / \mathrm{mL}$, respectively). As the oral dose increased eightfold (from 60 to $480 \mathrm{mg}$ ), mean $C_{\max }, \mathrm{AUC}_{0-\text { last }}$, and $\mathrm{AUC}_{0 \text {-inf }}$ increased 5.7-, 10.6-, and 6.4-fold, respectively. Median time to reach $C_{\max }\left(t_{\max }\right)$ following oral administration was short, ranging between 15 and $30 \mathrm{~min}$, independent of dose. After reaching $C_{\max }$, concentrations decreased rapidly until they were no longer quantifiable at $1.5-4 \mathrm{~h}$, depending on dose (Fig. $\mathrm{S} 2$ in the ESM). Plasma clearance was rapid, with a mean half-life $\left(t_{1 / 2}\right)$ ranging from 20 to $40 \mathrm{~min}$, and increased only slightly in relation to oral doses. Mean apparent oral clearance $(\mathrm{CL} / F)$ and apparent volume of distribution during the 
Table 1 Pharmacokinetic profile of oral and intravenous remimazolam and its metabolite (results of trial 1 and part 1 of trial 2)

\begin{tabular}{|c|c|c|c|c|c|c|c|}
\hline \multirow[t]{2}{*}{ PK parameter } & \multirow{2}{*}{$\begin{array}{l}\text { IV remimazolam } \\
1.25-2.58 \mathrm{mg} \\
(N=14)\end{array}$} & \multicolumn{6}{|l|}{ Oral remimazolam } \\
\hline & & $\begin{array}{l}7.00-14.4 \mathrm{mg} \\
(N=14)\end{array}$ & $\begin{array}{l}60 \mathrm{mg} \\
(N=3)\end{array}$ & $\begin{array}{l}140 \mathrm{mg} \\
(N=3)\end{array}$ & $\begin{array}{l}240 \mathrm{mg} \\
(N=5)\end{array}$ & $\begin{array}{l}360 \mathrm{mg} \\
(N=5)\end{array}$ & $\begin{array}{l}480 \mathrm{mg} \\
(N=5)\end{array}$ \\
\hline \multicolumn{8}{|l|}{$C_{\max }, \mathrm{ng} / \mathrm{mL}$} \\
\hline Remimazolam & $94.62 \pm 107.43$ & $4.55 \pm 1.61$ & $16.2 \pm 7.9$ & $36.9 \pm 7.1$ & $58.1 \pm 49.0$ & $60.9 \pm 32.0$ & $92.9 \pm 34.0$ \\
\hline CNS7054 & $118.91 \pm 29.51$ & $846.06 \pm 162.69$ & $4567 \pm 318$ & $13,597 \pm 1013$ & $25,390 \pm 3173$ & $30,558 \pm 5836$ & $41,876 \pm 10,198$ \\
\hline \multicolumn{8}{|c|}{$t_{\max }, \operatorname{median}(\min ; \max ), \mathrm{h}$} \\
\hline Remimazolam & $0.04(0.03 ; 0.10)$ & $0.50(0.08 ; 0.72)$ & $0.23(0.23 ; 0.73)$ & $0.48(0.23 ; 0.72)$ & $0.23(0.23 ; 0.75)$ & $0.48(0.23 ; 1.03)$ & $0.48(0.23 ; 0.73)$ \\
\hline CNS7054 & $0.50(0.25 ; 1.00)$ & $0.50(0.50 ; 1.00)$ & $0.73(0.47 ; 0.98)$ & $0.73(0.48 ; 0.97)$ & $0.73(0.48 ; 0.98)$ & $0.77(0.48 ; 1.53)$ & $1.03(0.75 ; 1.98)$ \\
\hline \multicolumn{8}{|l|}{$\mathrm{AUC}_{0-t}, \mathrm{~h} \cdot \mathrm{ng} / \mathrm{mL}$} \\
\hline Remimazolam & $21.78 \pm 10.01$ & $2.92 \pm 1.42$ & $11.1 \pm 6.5$ & $31.5 \pm 6.2$ & $43.8 \pm 35.0$ & $61.0 \pm 30.0$ & $118.0 \pm 57.0$ \\
\hline CNS7054 & $314.34 \pm 114.07$ & $1943.84 \pm 513.72$ & $9943 \pm 1259$ & $37,531 \pm 10,405$ & $61,189 \pm 15,578$ & $84,483 \pm 13,270$ & $122,285 \pm 27,012$ \\
\hline \multicolumn{8}{|c|}{$\mathrm{AUC}_{0-\text { inf }}, \mathrm{h} \cdot \mathrm{ng} / \mathrm{mL}$} \\
\hline Remimazolam & $24.03 \pm 10.72$ & $\mathrm{NC}$ & $19.5 \pm \mathrm{NC}$ & $35.7 \pm 8.1$ & $46.5 \pm 35.0$ & $64.3 \pm 30.0$ & $124.0 \pm 57.0$ \\
\hline CNS7054 & $474.23 \pm 78.61$ & $2088.28 \pm 569.89$ & $10,435 \pm 1342$ & $41,360 \pm 13,967$ & $65,315 \pm 18,690$ & $90,223 \pm 12,116$ & $12,6411 \pm 27,146$ \\
\hline \multicolumn{8}{|l|}{$t_{1 / 2}, \mathrm{~h}$} \\
\hline Remimazolam & $0.44 \pm 0.17$ & $\mathrm{NC}$ & $0.33 \pm \mathrm{NC}$ & $0.37 \pm 0.01$ & $0.55 \pm 0.17$ & $0.59 \pm 0.21$ & $0.69 \pm 0.25$ \\
\hline CNS7054 & $1.94 \pm 0.44$ & $2.54 \pm 0.57$ & $1.75 \pm 0.16$ & $2.09 \pm 0.45$ & $1.86 \pm 0.31$ & $1.96 \pm 0.35$ & $2.00 \pm 0.35$ \\
\hline \multicolumn{8}{|l|}{$\mathrm{CL} / F, \mathrm{~L} / \mathrm{h}$} \\
\hline Remimazolam & $89.60 \pm 29.81$ & $\mathrm{NC}$ & $3071 \pm \mathrm{NC}$ & $4024 \pm 916$ & $6724 \pm 2619$ & $7270 \pm 4826$ & $4428 \pm 1686$ \\
\hline CNS7054 & $\mathrm{NC}$ & $\mathrm{NC}$ & $\mathrm{NC}$ & $\mathrm{NC}$ & $\mathrm{NC}$ & $\mathrm{NC}$ & $\mathrm{NC}$ \\
\hline \multicolumn{8}{|l|}{$V_{z} / F, \mathrm{~L}$} \\
\hline Remimazolam & $54.10 \pm 22.38$ & $\mathrm{NC}$ & $1479 \pm \mathrm{NC}$ & $2140 \pm 543$ & $5202 \pm 2537$ & $6180 \pm 4056$ & $4448 \pm 2250$ \\
\hline CNS7054 & $\mathrm{NC}$ & $\mathrm{NC}$ & $\mathrm{NC}$ & $\mathrm{NC}$ & $\mathrm{NC}$ & $\mathrm{NC}$ & $\mathrm{NC}$ \\
\hline
\end{tabular}

Data are presented as mean \pm standard deviation unless otherwise indicated. $\mathrm{AUC}_{0-\text { inf }}, t_{1 / 2}, \mathrm{CL} / F$, and $V_{z} / F$ were not calculable when the percent extrapolation was $>20 \%$. Terminal phase parameters $\left(t_{1 / 2}, \mathrm{CL} / F\right.$, and $\left.V_{z} / F\right)$ were best characterized at doses of 240,360 , and $480 \mathrm{mg}$ with five subjects per dose level, whereas adequate data for these parameters were obtained in only one subject for the $60 \mathrm{mg}$ dose and two for the $140 \mathrm{mg}$ dose

$A U C$ area under the curve, $C L / F$ apparent oral clearance, $C_{\max }$ maximum observed plasma concentration, $I V$ intravenous, $N C$ not calculable, $P K$ pharmacokinetic, $t_{\max }$ time to reach $C_{\max }, t_{1 / 2}$ half-life, $V_{z} / F$ apparent volume of distribution

terminal phase $\left(V_{z} / F\right)$ all increased with oral doses and were markedly greater than with the intravenous dose.

For the metabolite CNS7054, $C_{\max }, \mathrm{AUC}_{0-\text { last }}$, and AUC 0 -inf were all considerably greater than those of the parent. These parameters also increased as the oral dose increased from 60 to $480 \mathrm{mg}$ by 9.2-, 12.3-, and 12.1-fold, respectively. Median $t_{\max }$ was short, ranging from 30 to $60 \mathrm{~min}$. For the higher oral doses of remimazolam $(60-480 \mathrm{mg})$, the $t_{\max }$ of the metabolite occurred only $15-30 \mathrm{~min}$ after the $t_{\max }$ of the parent. The mean half-life of the metabolite was greater than that of the parent, ranging from 1.75 to $2 \mathrm{~h}$, independent of dose.

\subsection{Pharmacokinetics of Oral Remimazolam with Alcohol}

Tables 2 and 3 and Fig. 1 present the pharmacokinetic results from part 2 of trial 2 (oral doses: remimazolam $360 \mathrm{mg}$, remimazolam $360 \mathrm{mg}+5 \% \mathrm{v} / \mathrm{v}$ alcohol, remimazolam $360 \mathrm{mg}+15 \% \mathrm{v} / \mathrm{v}$ alcohol, remimazolam $360 \mathrm{mg}+40 \% \mathrm{v} / \mathrm{v}$ alcohol, and placebo $+40 \% \mathrm{v} / \mathrm{v}$ alcohol). Table 2 presents the descriptive statistics and Table 3 the statistical analysis.

Alcohol increased the exposure to remimazolam. As the amount of alcohol increased from no alcohol to 5\%, $15 \%$, and $40 \%$, the $C_{\max }$ of remimazolam increased 1.2-, 1.5-, and 2.1-fold, respectively. Over the same dose range, $\mathrm{AUC}_{0-\text { last }}$ increased 1.1-, 1.6-, and 2.0-fold, and $\mathrm{AUC}_{0-\text { inf }}$ increased 1.1-, 1.6-, and 1.9-fold, respectively (Table 3). The median $t_{\max }$ was short, ranging between 15 and $30 \mathrm{~min}$, independent of the alcohol dose, and was similar to that obtained with remimazolam doses of $60-480 \mathrm{mg}$ without alcohol (results of part 1). After reaching $C_{\max }$, concentrations decreased rapidly until they were no longer quantifiable at $4 \mathrm{~h}$ (Fig. S2 in the ESM). Plasma clearance of remimazolam was also rapid, with mean $t_{1 / 2}$ ranging from 30 to $40 \mathrm{~min}$ and independent of alcohol dose. 

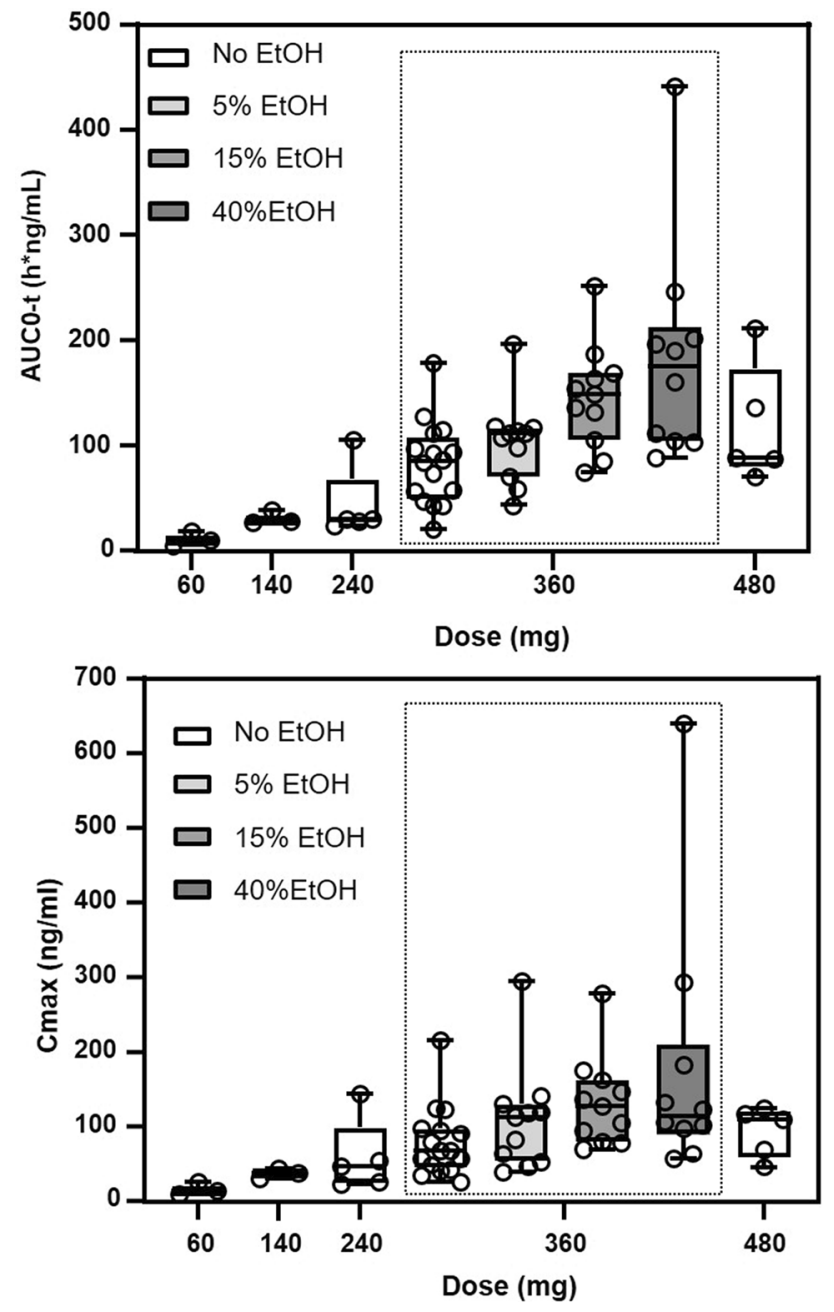

Fig. 1 Box and whisker plot including individual value plot of remimazolam $\mathrm{AUC}_{0-t}$ and $C_{\max }$ versus remimazolam and alcohol dose (results of trial 2). Box plots show 25-75 percentile and median values. Whisker plots indicate min and max values. $A U C$ area under the curve, $C_{\max }$ maximum observed plasma concentration, $\mathrm{EtOH}$ alcohol

However, the CL/F and $V_{z} / F$ of remimazolam decreased as the amount of alcohol increased.

The mean $C_{\max }, \mathrm{AUC}_{0-t}$, and $\mathrm{AUC}_{0 \text {-inf }}$ of the metabolite increased relative to the amount of added alcohol, but the degree of change was very modest. As co-administered alcohol concentrations increased from 0 to $5 \%, 15 \%$, and $40 \%$, $C_{\max }$ increased by 1.0-, 1.1-, and 1.1-fold; $\mathrm{AUC}_{0-t}$ increased by 1.0-, 1.1-, and 1.2-fold; and $\mathrm{AUC}_{0-\text { inf }}$ increased by 1.0-, $1.1-$, and 1.3-fold, respectively. Median $t_{\max }$ was approximately $1 \mathrm{~h}$ with $0 \% \mathrm{v} / \mathrm{v}$ or $5 \% \mathrm{v} / \mathrm{v}$ alcohol and increased to $1.5 \mathrm{~h}$ with $15 \% \mathrm{v} / \mathrm{v}$ or $40 \% \mathrm{v} / \mathrm{v}$ alcohol. Median $t_{\max }$ for CNS7054 occurred after approximately 30-60 min after the $t_{\max }$ for the parent. The half-life of the metabolite (1.86-2.09 h) was also somewhat greater than that of remimazolam and independent of the amount of alcohol.

\subsection{Oral Bioavailability of Remimazolam}

The absolute bioavailability of oral remimazolam was very low: $2.2 \%$ (90\% CI 0.015-0.032) based on $\mathrm{AUC}_{0-t}$ and 1.2\% (90\% CI 0.008-0.017) based on $C_{\max }$ (Table 4). In contrast, the oral bioavailability of the metabolite $(>100 \%)$ was considerably greater than that of the parent drug, indicating that low oral bioavailability of remimazolam was an effect of extensive first-pass metabolism and not of low absorption.

\subsection{Pharmacodynamics of Oral Remimazolam}

Table 5 presents the results of the pharmacodynamic assessments from part 1 of trial 2 with five oral remimazolam doses tested: 60, 140, 240, 360, and $480 \mathrm{mg}$. No pharmacodynamic assessments were performed in trial 1 .

Mean alertness/drowsiness VAS minimum effect $\left(E_{\min }\right)$ showed a mild dose-dependent drowsiness up to $240 \mathrm{mg}$, with a plateau at higher doses. Similarly, sedation was not achieved with the two lowest doses $(60$ and $140 \mathrm{mg}$ ) of remimazolam (MOAA/S $=5$ ), whereas one subject each treated with 240 and $360 \mathrm{mg}$ reported an MOAA/S score of 4 at $15 \mathrm{~min}$ postdose and one subject treated with $480 \mathrm{mg}$ reported an MOAA/S score of 3 at 45 min postdose. Results of the PAL and RTI 5-choice tests suggested that subjects' learning performance and reaction time were not affected by oral remimazolam.

As a result, an MBAD of oral remimazolam (defined as MOAA/S $<3$ in at least two of three subjects in the $60 / 140 \mathrm{mg}$ groups or at least three of five subjects in the $240 / 360 / 480 \mathrm{mg}$ groups) was not identified in this part of the trial.

\subsection{Pharmacodynamics of Oral Remimazolam with Alcohol}

Table 6 presents the pharmacodynamic results from part 2 of trial 2 (oral doses: remimazolam $360 \mathrm{mg}$, remimazolam $360 \mathrm{mg}+5 \% \mathrm{v} / \mathrm{v}$ alcohol, remimazolam $360 \mathrm{mg}+15 \% \mathrm{v} / \mathrm{v}$ alcohol, remimazolam $360 \mathrm{mg}+40 \% \mathrm{v} / \mathrm{v}$ alcohol, and placebo $+40 \%$ v/v alcohol).

Drowsiness (VAS $<50$ ) was observed in all five groups; the greatest alertness/drowsiness VAS $E_{\min }$ was in the placebo $+40 \% \mathrm{v} / \mathrm{v}$ alcohol group, followed by the remimazolam alone group and the three remimazolam + alcohol groups. No trend of alcohol dose dependency was observed. Mean MOAA/S scores of 4.13 or 4.50 indicated almost no sedative effects. One subject had an MOAA/S of 2 following treatment with remimazolam alone and an MOAA/S of 3 following treatment with remimazolam $+15 \% \mathrm{v} / \mathrm{v}$ alcohol. Another subject had an MOAA/S of 2 and an MOAA/S of 1 at two time points after remimazolam $+40 \% \mathrm{v} / \mathrm{v}$ alcohol. In line 
Table 2 Pharmacokinetic parameters for remimazolam and its metabolite in plasma after oral administration of remimazolam $360 \mathrm{mg}$ with and without alcohol (results of trial 2)

\begin{tabular}{|c|c|c|c|c|}
\hline PK parameter & $\begin{array}{l}\text { Remimazolam } 360 \mathrm{mg} \\
(N=11)\end{array}$ & $\begin{array}{l}\text { Remimazolam } 360 \mathrm{mg}+5 \% \mathrm{v} / \mathrm{v} \\
\text { alcohol }(N=11)\end{array}$ & $\begin{array}{l}\text { Remimazolam } 360 \mathrm{mg}+15 \% \\
\mathrm{v} / \mathrm{v} \text { alcohol }(N=11)\end{array}$ & $\begin{array}{l}\text { Remimazolam } \\
360 \mathrm{mg}+40 \% \mathrm{v} / \mathrm{v} \\
\text { alcohol }(N=8)\end{array}$ \\
\hline \multicolumn{5}{|l|}{$C_{\max }, \mathrm{ng} / \mathrm{mL}$} \\
\hline Remimazolam & $87.3 \pm 52.0$ & $109 \pm 71$ & $131 \pm 60$ & $180 \pm 189$ \\
\hline CNS7054 & $26,389 \pm 4925$ & $27,651 \pm 7977$ & $27,883 \pm 6062$ & $27,869 \pm 4792$ \\
\hline \multicolumn{5}{|c|}{$t_{\max }, \operatorname{median}(\min ; \max ), \mathrm{h}$} \\
\hline Remimazolam & $0.48(0.23 ; 0.98)$ & $0.48(0.22 ; 0.75)$ & $0.23(0.22 ; 0.97)$ & $0.49(0.18 ; 0.97)$ \\
\hline CNS7054 & $0.98(0.73 ; 1.98)$ & $0.97(0.47 ; 3.03)$ & $1.48(0.48 ; 3.02)$ & $1.49(0.48 ; 3.02)$ \\
\hline \multicolumn{5}{|l|}{$\mathrm{AUC}_{0-t}, \mathrm{~h} \cdot \mathrm{ng} / \mathrm{mL}$} \\
\hline Remimazolam & $93.0 \pm 40.0$ & $104 \pm 40$ & $146 \pm 50$ & $185 \pm 113$ \\
\hline CNS7054 & $83,313 \pm 13,189$ & $84,361 \pm 15,011$ & $90,367 \pm 18,276$ & $102,031 \pm 22,152$ \\
\hline \multicolumn{5}{|c|}{$\mathrm{AUC}_{0-\mathrm{inf}}, \mathrm{h} \cdot \mathrm{ng} / \mathrm{mL}$} \\
\hline Remimazolam & $97.6 \pm 39$ & $108 \pm 39$ & $152 \pm 51$ & $190 \pm 113$ \\
\hline CNS7054 & $89,041 \pm 13,312$ & $91,309 \pm 15,733$ & $97,102 \pm 20,796$ & $112,894 \pm 24,734$ \\
\hline \multicolumn{5}{|l|}{$t_{1 / 2}, \mathrm{~h}$} \\
\hline Remimazolam & $0.61 \pm 0.15$ & $0.65 \pm 0.18$ & $0.56 \pm 0.12$ & $0.55 \pm 0.10$ \\
\hline CNS7054 & $1.86 \pm 0.45$ & $2.00 \pm 0.26$ & $1.90 \pm 0.44$ & $2.09 \pm 0.61$ \\
\hline \multicolumn{5}{|l|}{$\mathrm{CL} / F, \mathrm{~L} / \mathrm{h}$} \\
\hline Remimazolam & $4325 \pm 1896$ & $3773 \pm 1531$ & $2625 \pm 933$ & $2366 \pm 1031$ \\
\hline CNS7054 & $\mathrm{NC}$ & $\mathrm{NC}$ & $\mathrm{NC}$ & $\mathrm{NC}$ \\
\hline \multicolumn{5}{|l|}{$V_{z} / F, \mathrm{~L}$} \\
\hline Remimazolam & $3692 \pm 1668$ & $3548 \pm 1826$ & $2207 \pm 1086$ & $1796 \pm 756$ \\
\hline CNS7054 & $\mathrm{NC}$ & $\mathrm{NC}$ & $\mathrm{NC}$ & $\mathrm{NC}$ \\
\hline
\end{tabular}

$\mathrm{AUC}_{0 \text {-inf }}, t_{1 / 2}, \mathrm{CL} / F$, and $V_{z} / F$ were not calculable when the percent extrapolation was $>20 \%$

$A U C$ area under the curve, $C L / F$ apparent oral clearance, $C_{\max }$ maximum observed plasma concentration, $I V$ intravenous, $N C$ not calculable, $P K$ pharmacokinetic, $t_{1 / 2}$ half-life, $t_{\max }$ time to reach $C_{\max }, V_{z} / F$ apparent volume of distribution

Table 3 Statistical analysis of the effect of alcohol on systemic exposure to remimazolam and CNS7054

\begin{tabular}{llll}
\hline Test vs. reference & PK parameter & \multicolumn{2}{l}{ LS geometric mean ratio (90\% CI) } \\
\cline { 3 - 4 } & & Remimazolam & CNS7054 \\
\hline Remimazolam $360 \mathrm{mg}+5 \%$ v/v alcohol vs. remimazolam $360 \mathrm{mg}$ alone & $C_{\max }(\mathrm{ng} / \mathrm{mL})$ & $1.195(0.983-1.452)$ & $1.019(0.913-1.138)$ \\
& $\mathrm{AUC}_{0-\text { last }}(\mathrm{h} \cdot \mathrm{ng} / \mathrm{mL})$ & $1.127(1.039-1.223)$ & $1.005(0.949-1.065)$ \\
& $\mathrm{AUC}_{0-\text {-inf }}(\mathrm{h} \cdot \mathrm{ng} / \mathrm{mL})$ & $1.116(1.030-1.209)$ & $1.019(0.954-1.088)$ \\
Remimazolam $360 \mathrm{mg}+15 \% \mathrm{v} / \mathrm{v}$ alcohol vs. remimazolam $360 \mathrm{mg}$ alone & $C_{\max }(\mathrm{ng} / \mathrm{mL})$ & $1.572(1.294-1.911)$ & $1.035(0.927-1.156)$ \\
& $\mathrm{AUC}_{0-\mathrm{last}}(\mathrm{h} \cdot \mathrm{ng} / \mathrm{mL})$ & $1.617(1.490-1.754)$ & $1.066(1.007-1.130)$ \\
& $\mathrm{AUC}_{0-\mathrm{inf}}(\mathrm{h} \cdot \mathrm{ng} / \mathrm{mL})$ & $1.593(1.467-1.730)$ & $1.066(0.998-1.138)$ \\
Remimazolam $360 \mathrm{mg}+40 \% \mathrm{v} / \mathrm{v}$ alcohol vs. remimazolam $360 \mathrm{mg}$ alone & $C_{\text {max }}(\mathrm{ng} / \mathrm{mL})$ & $1.866(1.500-2.323)$ & $1.058(0.936-1.197)$ \\
& $\mathrm{AUC}_{0-\text { last }}(\mathrm{h} \cdot \mathrm{ng} / \mathrm{mL})$ & $2.073(1.892-2.272)$ & $1.195(1.120-1.275)$ \\
& $\mathrm{AUC}_{0-\text {-inf }}(\mathrm{h} \cdot \mathrm{ng} / \mathrm{mL})$ & $1.995(1.823-2.183)$ & $1.225(1.138-1.319)$ \\
\hline
\end{tabular}

$A U C$ area under the curve, $C I$ confidence interval, $C_{\max }$ maximum observed plasma concentration, $L S$ least squares, $P K$ pharmacokinetic

with these results, subjects' learning performance seemed to be slightly worse in the remimazolam + alcohol groups than in the remimazolam alone or alcohol alone groups. However, the reaction time was not increased when remimazolam was co-ingested with alcohol.

\subsection{Safety}

There were no fatal, serious, or severe adverse events, nor did any adverse events lead to discontinuation of study drug in either trial. Almost all subjects reported at least one treatment-emergent adverse event (12/14 [85.7\%] in trial 1, 19/21 
Table 4 Oral bioavailability of remimazolam and its metabolite

\begin{tabular}{llll}
\hline Analyte & Parameter & Bioavailability & $90 \%$ CI \\
\hline Remimazolam & $C_{\max } / D$ & 0.012 & $0.008-0.017$ \\
& $\mathrm{AUC}_{0-t} / D$ & 0.022 & $0.015-0.032$ \\
CNS7054 & $C_{\max } / D$ & 1.283 & $1.183-1.390$ \\
& $\mathrm{AUC}_{0-t} / D$ & 1.149 & $1.031-1.280$ \\
\hline
\end{tabular}

$A U C$ area under the curve, $C I$ confidence interval, $C_{\max }$ maximum observed plasma concentration

[90.5\%] in part 1 of trial 2, 11/11 [100\%] in part 2 of trial 2). Most of these were transient and of mild severity.

Adverse events of interest in trial 1 are presented in Table S1 in the ESM, and abuse-related adverse events in trial 2 are presented in Table S2 and Table S3 in the ESM. In general, more adverse events were reported in part 2 of trial 2 than in trial 1 or part 1 of trial 2 . The most common adverse event was somnolence $(21.4 \%$ in trial $1,47.6 \%$ in part 1 of trial 2, 100\% in part 2 of trial 2). All somnolence adverse events were of mild severity in trial 1 and part 1 of trial 2, whereas about $50 \%$ were of moderate severity in part 2 of trial 2, in line with the observed additive effects between remimazolam and alcohol.

There were no important changes in laboratory parameters in any trial. No clinically important changes in vital signs were reported in trial 1 . In both parts of trial 2, although clinically important changes in vital signs were observed in some subjects, there were no meaningful or remimazolam/alcohol dose-dependent trends in blood pressure, heart rate, respiratory rate, or blood oxygen saturation changes. No subjects required airway intervention.

\section{Discussion}

The pharmacokinetic results of trial 1 and part 1 of trial 2 indicate that remimazolam is completely absorbed from the gastrointestinal system but then undergoes rapid and extensive first-pass metabolism following oral administration, regardless of dose level, resulting in a markedly low oral bioavailability (2.2\% based on $\mathrm{AUC}_{0-t}$ and $1.2 \%$ based on $C_{\text {max }}$ ). The oral bioavailability of remimazolam can be considered negligible compared with that of other benzodiazepines (midazolam 31-72\% [16], flunitrazepam 50\% [17], diazepam > 90\% [18], and lorazepam 91-95\% [19]).

Oral remimazolam is metabolized and absorbed considerably faster than other benzodiazepines: $t_{1 / 2}$ remimazolam $0.3-0.7 \mathrm{~h}$ versus midazolam $1.7-3.5 \mathrm{~h}$, lorazepam $8-15 \mathrm{~h}$, diazepam $30 \mathrm{~h}$, and flunitrazepam $15-30 \mathrm{~h} ; t_{\max }$ remimazolam 15-30 min versus midazolam 30-80 $\mathrm{min}$, lorazepam $2 \mathrm{~h}$, diazepam $1 \mathrm{~h}$, and flunitrazepam $0.6 \mathrm{~h}$ [20-22]. Another study indicated that the elimination half-life of short-acting benzodiazepines ranges between 1 and $12 \mathrm{~h}$ following oral administration [10], making remimazolam ultra short acting. Indeed, remimazolam was designed to undergo rapid hydrolysis by esterase enzymes (mainly localized in the liver), thereby offering more rapid and predictable onset and offset of action than other available benzodiazepines [1].

Of note, the pharmacokinetic results of part 2 of trial 2 demonstrated that alcohol did not inhibit the first pass
Table 5 Selected descriptive statistics for main pharmacodynamic parameters of oral remimazolam (results of part 1 of trial 2)

\begin{tabular}{|c|c|c|c|c|c|}
\hline PD parameter & $60 \mathrm{mg}(N=3)$ & $140 \mathrm{mg}(N=3)$ & $240 \mathrm{mg}(N=5)$ & $360 \mathrm{mg}(N=5)$ & $480 \mathrm{mg}(N=5)$ \\
\hline \multicolumn{6}{|c|}{ Alertness/drowsiness VAS $\left(E_{\min }\right)$} \\
\hline Mean & 60.7 & 47.0 & 38.0 & 37.0 & 36.4 \\
\hline SD & 15 & 1.0 & 16 & 5.8 & 9.3 \\
\hline Median & 69.0 & 47.0 & 39.0 & 39.0 & 41.0 \\
\hline \multicolumn{6}{|l|}{$\mathrm{MOAA} / \mathrm{S}\left(E_{\min }\right)$} \\
\hline Mean & 5.0 & 5.0 & 4.8 & 4.8 & 4.6 \\
\hline SD & 0 & 0 & 0.45 & 0.45 & 0.89 \\
\hline Median & 5.0 & 5.0 & 5.0 & 5.0 & 5.0 \\
\hline \multicolumn{6}{|c|}{ PAL total errors (adjusted, $E_{\max }$ ) } \\
\hline Mean & 5.67 & 17.3 & 22.8 & 11.2 & 12.6 \\
\hline SD & 0.58 & 22 & 20 & 3.3 & 5.3 \\
\hline Median & 6.0 & 8.0 & 14 & 11.0 & 12.0 \\
\hline \multicolumn{6}{|c|}{ RTI 5-choice reaction time: median $\left(E_{\max }, \mathrm{ms}\right)$} \\
\hline Mean & 418 & 459 & 479 & 452 & 435 \\
\hline SD & 27 & 88 & 54 & 36 & 22 \\
\hline Median & 404 & 416 & 483 & 452 & 436 \\
\hline
\end{tabular}

$E_{\text {max }}$ maximum effect, $E_{\text {min }}$ minimum effect, $M O A A / S$ modified observer's assessment of alertness/sedation, $P A L$ paired associated learning test, $P D$ pharmacodynamic, $R T I$ reaction time test, $S D$ standard deviation, VAS visual analogue scale 
Table 6 Selected statistics for main pharmacodynamic parameter endpoints of oral remimazolam $360 \mathrm{mg}$ co-administered with alcohol (results of part 2 of trial 2)

\begin{tabular}{|c|c|c|c|c|c|}
\hline PD parameter & $\begin{array}{l}\text { Remimazolam } 360 \mathrm{mg} \\
(N=10)\end{array}$ & $\begin{array}{l}\text { Remimazolam } \\
360 \mathrm{mg}+5 \% \mathrm{v} / \mathrm{v} \text { alcohol } \\
(N=10)\end{array}$ & $\begin{array}{l}\text { Remimazolam } \\
360 \mathrm{mg}+15 \% \mathrm{v} / \mathrm{v} \\
\text { alcohol }(N=10)\end{array}$ & $\begin{array}{l}\text { Remimazolam } \\
360 \mathrm{mg}+40 \% \mathrm{v} / \mathrm{v} \\
\text { alcohol }(N=8)\end{array}$ & $\begin{array}{l}\text { Placebo }+40 \% \\
\text { v/v alcohol } \\
(N=10)\end{array}$ \\
\hline \multicolumn{6}{|c|}{ Alertness/drowsiness VAS $\left(E_{\min }\right)$} \\
\hline Mean \pm SD & $30.7 \pm 17$ & $23.9 \pm 16$ & $25.6 \pm 12$ & $26.5 \pm 16$ & $41.6 \pm 17$ \\
\hline Median & 32.5 & 21.0 & 22.0 & 25.0 & 42.0 \\
\hline \multicolumn{6}{|l|}{$\begin{array}{l}\text { LS mean difference } \\
\quad(90 \% \mathrm{CI})\end{array}$} \\
\hline vs. remimazolam & NA & $-7.3(-19.3$ to 4.7$)$ & $-4.8(-16.7-7.12)$ & $-3.8(-16.5-8.8)$ & NA \\
\hline vs. $40 \%$ alcohol & $-12.2(-24.2$ to -0.2$)$ & $\begin{array}{l}-19.5(-31.45 \text { to } \\
-7.6)\end{array}$ & $\begin{array}{l}-17.0(-29.0 \text { to } \\
-5.1)\end{array}$ & $\begin{array}{l}-16.1(-28.7 \text { to } \\
-3.4)\end{array}$ & NA \\
\hline \multicolumn{6}{|l|}{$\mathrm{MOAA} / \mathrm{S}\left(E_{\min }\right)$} \\
\hline Mean \pm SD & $4.50 \pm 0.97$ & $4.50 \pm 0.53$ & $4.50 \pm 0.71$ & $4.13 \pm 1.4$ & $5.00 \pm 0$ \\
\hline Median & 5.00 & 4.50 & 5.00 & 4.50 & 5.00 \\
\hline \multicolumn{6}{|l|}{$\begin{array}{l}\text { LS mean difference } \\
\quad(90 \% \mathrm{CI})\end{array}$} \\
\hline vs. remimazolam & NA & $0.12(-0.37-0.61)$ & $-0.01(-0.50-0.47)$ & $\begin{array}{l}-0.46(-0.98- \\
0.06)\end{array}$ & NA \\
\hline vs. $40 \%$ alcohol & $\begin{array}{l}-0.58(-1.07 \text { to } \\
-0.09)\end{array}$ & $-0.45(-0.94-0.03)$ & $\begin{array}{l}-0.59(-1.08 \text { to } \\
-0.10)\end{array}$ & $\begin{array}{l}-1.03(-1.56 \text { to } \\
-0.51)\end{array}$ & NA \\
\hline \multicolumn{6}{|c|}{ PAL total errors (adjusted, $E_{\max }$ ) } \\
\hline Mean \pm SD & $20.4 \pm 17$ & $33.2 \pm 26$ & $31.0 \pm 25$ & $29.0 \pm 19$ & $15.5 \pm 11$ \\
\hline Median & 19.5 & 34.5 & 17.0 & 21.0 & 13.5 \\
\hline \multicolumn{6}{|l|}{$\begin{array}{l}\text { LS mean difference } \\
\quad(90 \% \mathrm{CI})\end{array}$} \\
\hline vs. remimazolam & NA & $12.3(1.7-22.9)$ & $7.3(-3.2-17.8)$ & $8.0(-3.3-19.3)$ & NA \\
\hline vs. $40 \%$ alcohol & $6.19(-4.4-16.8)$ & $18.5(7.9-29.0)$ & $13.5(3.0-24.0)$ & $14.2(2.9-25.5)$ & NA \\
\hline \multicolumn{6}{|c|}{ RTI 5-choice reaction time: median $\left(E_{\max }[\mathrm{ms}]\right)$} \\
\hline Mean \pm SD & $496 \pm 75$ & $473 \pm 55$ & $509 \pm 54$ & $492 \pm 45$ & $461 \pm 52$ \\
\hline Median & 483 & 469 & 502 & 479 & 453 \\
\hline \multicolumn{6}{|l|}{$\begin{array}{l}\text { LS mean difference } \\
\quad(90 \% \mathrm{CI})\end{array}$} \\
\hline vs. remimazolam & NA & $-27.63(-56.48-1.21)$ & $4.76(-23.94-33.45)$ & $\begin{array}{l}-6.53(-37.28- \\
24.22)\end{array}$ & NA \\
\hline vs. $40 \%$ alcohol & $39.8(11.0-68.6)$ & $12.2(-16.5-40.9)$ & $44.6(15.9-73.3)$ & $\begin{array}{l}33.27(2.53- \\
64.01)\end{array}$ & NA \\
\hline
\end{tabular}

The $E_{\max }$ and $E_{\min }$ analyses were performed using a linear mixed-effects model with treatment and period as fixed effects and subject as a random effect

$C I$ confidence interval, $E_{\max }$ maximum effect, $E_{\min }$ minimum effect, $L S$ least squares, $M O A A / S$ modified observer's assessment of alertness/sedation, $N A$ not applicable, $P A L$ paired associated learning test, $P D$ pharmacodynamic, $R T I$ reaction time test, $S D$ standard deviation, $V A S$ visual analogue scale

metabolism of remimazolam, even though remimazolam systemic exposure increased proportionally with the coingested alcohol amount, and it doubled after remimazolam $360 \mathrm{mg}+40 \mathrm{v} / \mathrm{v}$ alcohol, consistent with the known effect of alcohol as an inhibitor of CES1. Nevertheless, remimazolam was still extensively metabolized and disappeared from plasma rapidly, even in the presence of alcohol. Independent of alcohol dose, mean $t_{1 / 2}$ ranged from 20 to $40 \mathrm{~min}$ and median $t_{\max }$ from 15 to $30 \mathrm{~min}$, similar to observations at remimazolam $60-480 \mathrm{mg}$ doses without alcohol. Only the formation of the pharmacologically inactive metabolite CNS7054 appeared to be delayed slightly, as the median $t_{\max }$ extended to $1-1.5 \mathrm{~h}$, but the $t_{1 / 2}$ of $1.86-2.09 \mathrm{~h}$ was comparable to the $1.75-2 \mathrm{~h}$ for the $60-480 \mathrm{mg}$ doses without alcohol, indicating that alcohol has little to no impact on elimination of the metabolite. Given the negligible oral bioavailability of remimazolam, alcohol-induced increases in exposure can still be considered insignificant, as one can speculate minor increases in oral bioavailability (two times, from approximately $1-2 \%$ to approximately $2-4 \%$ ). 
Interpretation of these results suffers to some extent from the need to use the bioavailability results from trial 1 , where different doses were used, as no intravenous arm was present in trial 2. Nevertheless, these extrapolations could be considered conservative, as bioavailability was more likely to go down with increasing oral doses in trial 2.

In line with its pharmacokinetic profile, the sedative effects of oral remimazolam are also limited. In part 1 of trial 2, even the highest oral dose of remimazolam $(480 \mathrm{mg})$ did not appear to impair subjects' alertness, learning performance, or reaction time. These results are in line with the recently published PK/PD model [23], where the halfmaximal effective concentration for an MOAA/S score of 4 was approximately threefold higher than the $C_{\max }$ reported in this trial. The intravenous dose used in the phase III clinical trials for induction of procedural sedation is $5 \mathrm{mg}$, almost 100 -fold lower. In part 2 of trial 2, even though the systemic exposure to remimazolam was increased in the presence of alcohol, changes in pharmacodynamic sedative effects were modest compared with remimazolam alone. Only one of ten female subjects attained a significant level of sedation (i.e. MOAA/S of 1). As such, these pharmacodynamic effects were not adequately predictable and reliable for use in drugfacilitated sexual assaults.

The remimazolam drug product for intravenous administration is supplied in $20 \mathrm{mg}$ vials; therefore, to achieve the dose level tested in part 2 of trial $2(360 \mathrm{mg})$, as many as 18 vials of trial drug would be needed. Importantly, remimazolam has a distinct bitter taste, which is difficult to mask and would make it unsuitable for covert use in drug- facilitated sexual assaults.

\section{Conclusions}

Collectively, the oral bioavailability of remimazolam is negligible and the pharmacodynamic assessment of alcohol and remimazolam combinations shows no potential for its use in drug-facilitated sexual assaults.

Author Contributions Conceptualization: MP, TS, KB, MD, and FS. Methodology: MP, TS, KB, LW, and FS. Formal analysis and investigation: JO. Original draft preparation: V-AD. Review and editing of manuscript: MP, TS, KB, V-AD, LW, and FS.

\section{Compliance with Ethical Standards}

Funding This work was sponsored by PAION UK Ltd (Cambridge, UK).

Conflict of interest Marija Pesic, Thomas Stöhr, Keith Borkett, Martin Donsbach, Van-Anh Dao, and Frank Schippers, as current or former employees of PAION, may own stocks or stock options of PAION.
Lynn Webster is an employee at PRA Early Development Services, the CRO that conducted these two clinical trials.

Ethics approval Both trials were carried out in accordance with The Code of Ethics of the World Medical Association (Declaration of Helsinki). Trial 1 protocol was approved by the Midlands Independent Review Board and trial 2 protocol was approved by the Schulman Independent Review Board.

Consent to participate Informed consent was obtained from all participants included in the study.

Consent for publication Participants signed informed consent regarding publishing their data.

Availability of data and material The datasets generated during and/ or analysed during the current study are not publicly available because of confidentiality restraints but are available from the corresponding author on reasonable request.

Open Access This article is licensed under a Creative Commons Attribution-NonCommercial 4.0 International License, which permits any non-commercial use, sharing, adaptation, distribution and reproduction in any medium or format, as long as you give appropriate credit to the original author(s) and the source, provide a link to the Creative Commons licence, and indicate if changes were made. The images or other third party material in this article are included in the article's Creative Commons licence, unless indicated otherwise in a credit line to the material. If material is not included in the article's Creative Commons licence and your intended use is not permitted by statutory regulation or exceeds the permitted use, you will need to obtain permission directly from the copyright holder. To view a copy of this licence, visit http://creativecommons.org/licenses/by-nc/4.0/.

\section{References}

1. Bansal S, Singhal S. Remimazolam (CNS7056): an Emerging Sedative and General Anaesthetic. J Clin Diagn Res. 2018;12(3):UE01-03. https://doi.org/10.7860/JCDR/2018/30823 .11263 .

2. Wittenborn JR. Effects of benzodiazepines on psychomotor performance. Br J Clin Pharmacol. 1979;7(Suppl 1):61S-7S. https ://doi.org/10.1111/j.1365-2125.1979.tb04667.x.

3. Testa M, Livingston JA, Vanzile-Tamsen C, Frone MR. The role of women's substance use in vulnerability to forcible and incapacitated rape. J Stud Alcohol. 2003;64(6):756-64. https://doi. org/10.15288/jsa.2003.64.756.

4. Fiorentin TR, Logan BK. Toxicological findings in 1000 cases of suspected drug facilitated sexual assault in the United States. J Forensic Leg Med. 2019;61:56-64. https://doi.org/10.1016/j. jflm.2018.11.006.

5. Du Mont J, Macdonald S, Rotbard N, Asllani E, Bainbridge D, Cohen MM. Factors associated with suspected drug-facilitated sexual assault. CMAJ. 2009;180(5):513-9. https://doi. org/10.1503/cmaj.080570.

6. Grela A, Gautam L, Cole MD. A multifactorial critical appraisal of substances found in drug facilitated sexual assault cases. Forensic Sci Int. 2018;292:50-60. https://doi.org/10.1016/j.forsc iint.2018.08.034.

7. Baldwin DS, Aitchison K, Bateson A, Curran HV, Davies $\mathrm{S}$, Leonard B, et al. Benzodiazepines: risks and benefits. A 
reconsideration. J Psychopharmacol. 2013;27(11):967-71. https ://doi.org/10.1177/0269881113503509.

8. Seppala T, Nuotto E, Dreyfus JF. Drug-alcohol interactions on psychomotor skills: zopiclone and flunitrazepam. Pharmacology. 1983;27(Suppl 2):127-35. https://doi.org/10.1159/000137919.

9. Tanaka E, Misawa S. Pharmacokinetic interactions between acute alcohol ingestion and single doses of benzodiazepines, and tricyclic and tetracyclic antidepressants-an update. J Clin Pharm Ther. 1998;23(5):331-6.

10. Griffin CE 3rd, Kaye AM, Bueno FR, Kaye AD. Benzodiazepine pharmacology and central nervous system-mediated effects. Ochsner J. 2013;13(2):214-23.

11. Leary A, MacDonald T. Interactions between alcohol and drugs. Proc R Coll Physicians Edinb. 1999;29(137):43.

12. Parker RB, Hu ZY, Meibohm B, Laizure SC. Effects of alcohol on human carboxylesterase drug metabolism. Clin Pharmacokinet. 2015;54(6):627-38. https://doi.org/10.1007/s40262-014-0226-2.

13. Antonik LJ, Goldwater DR, Kilpatrick GJ, Tilbrook GS, Borkett KM. A placebo- and midazolam-controlled phase I single ascending-dose study evaluating the safety, pharmacokinetics, and pharmacodynamics of remimazolam (CNS 7056): Part I. Safety, efficacy, and basic pharmacokinetics. Anesth Analg. 2012;115(2):274-83. https://doi.org/10.1213/ANE.0b013e3182 $3 \mathrm{f0c} 28$.

14. Rusted JM, Warburton DM. The effects of scopolamine on working memory in healthy young volunteers. Psychopharmacology. 1988;96(2):145-52.

15. Sahakian BJ, Morris RG, Evenden JL, Heald A, Levy R, Philpot $\mathrm{M}$, et al. A comparative study of visuospatial memory and learning in Alzheimer-type dementia and Parkinson's disease. Brain. 1988;111(Pt 3):695-718. https://doi.org/10.1093/brain/111.3.695.
16. Heizmann P, Eckert M, Ziegler WH. Pharmacokinetics and bioavailability of midazolam in man. Br J Clin Pharmacol. 1983;16(Suppl 1):43S-9S. https://doi. org/10.1111/j.1365-2125.1983.tb02270.x.

17. Cano JP, Soliva M, Hartmann D, Ziegler WH, Amrein R. Bioavailability from various galenic formulations of flunitrazepam. Arzneimittelforschung. 1977;27(12):2383-8.

18. Divoll M, Greenblatt DJ, Ochs HR, Shader RI. Absolute bioavailability of oral and intramuscular diazepam: effects of age and sex. Anesth Analg. 1983;62(1):1-8.

19. Greenblatt DJ, Shader RI, Franke K, MacLaughlin DS, Harmatz JS, Allen MD, et al. Pharmacokinetics and bioavailability of intravenous, intramuscular, and oral lorazepam in humans. J Pharm Sci. 1979;68(1):57-63. https://doi.org/10.1002/jps.2600680119.

20. Saari TI, Uusi-Oukari M, Ahonen J, Olkkola KT. Enhancement of GABAergic activity: neuropharmacological effects of benzodiazepines and therapeutic use in anesthesiology. Pharmacol Rev. 2011;63(1):243-67. https://doi.org/10.1124/pr.110.002717.

21. Breimer DD. Pharmacokinetics and metabolism of various benzodiazepines used as hypnotics. Br J Clin Pharmacol. 1979;8(1):7S13S. https://doi.org/10.1111/j.1365-2125.1979.tb00449.x.

22. Seppala M, Alihanka J, Himberg JJ, Kanto J, Rajala T, Sourander L. Midazolam and flunitrazepam: pharmacokinetics and effects on night time respiration and body movements in the elderly. Int J Clin Pharmacol Ther Toxicol. 1993;31(4):170-6.

23. Schuttler J, Eisenried A, Lerch M, Fechner J, Jeleazcov C, Ihmsen $\mathrm{H}$. Pharmacokinetics and pharmacodynamics of remimazolam (CNS 7056) after continuous infusion in healthy male volunteers: part I. Pharmacokinetics and clinical pharmacodynamics. Anesthesiology. 2020;132(4):636-51. https://doi.org/10.1097/ ALN.0000000000003103. 